\title{
Designers' Utilization of and Requirements on Design for Environment (DfE) Methods and Tools
}

\author{
Mattias Lindahl \\ Linköping University Post Print
}

Tweet

N.B.: When citing this work, cite the original article.

Original Publication:

Mattias Lindahl, Designers' Utilization of and Requirements on Design for Environment (DfE) Methods and Tools, 2005, Fourth International Symposium on Environmentally Conscious Design and Inverse Manufacturing, 2005. Eco Design 2005, 224-231. ISBN: 1-4244-0081-3

http://dx.doi.org/10.1109/ECODIM.2005.1619207

From the Fourth International Symposium on Environmentally Conscious Design and Inverse Manufacturing (Eco Design 2005), Tokyo, Japan, 12-14 December 2005

(C) 2005 IEEE. Personal use of this material is permitted. Permission from IEEE must be obtained for all other uses, in any current or future media, including reprinting/republishing this material for advertising or promotional purposes, creating new collective works, for resale or redistribution to servers or lists, or reuse of any copyrighted component of this work in other works.

Postprint available at: Linköping University Electronic Press

http://urn.kb.se/resolve?urn=urn:nbn:se:liu:diva-29933 


\title{
Designers' Utilization of and Requirements on Design for Environment (DfE) Methods and Tools
}

\author{
Mattias Lindahl \\ Environmental Technology and Management, Department of Mechanical Engineering, \\ Linköping University, Sweden \\ Mattias.Lindahl@ikp.liu.se
}

\begin{abstract}
The objectives of this paper are twofold: to identify engineering designers utilization of Design for Environment (DfE) methods and tools, and to investigate what basic design-related requirements a DfE method or tool should fulfill in order to become actively used in industry among engineering designers.

Most of the requirements for designers are related to their aims of fulfilling product performance and minimizing development time. There are four major requirements that a DfE method or tool, as well as a common method or tool, must exhibit. First, it must be easy to adopt and implement; second, it must facilitate designers to fulfill specified requirements on the presumptive product. Third, it must reduce the risk that important elements in the product development phase are forgotten. The two latter requirements relate to a method or tool's degree of appropriateness, but also to the fourth requirement, which is considered here the most important: that the use of the method or tool must reduce the total calendar time (from start to end) to solve the task. The conclusion is that DfE methods and tools must be designed to better comply with its main users - in this case the designers.
\end{abstract}

Key words: Design for Environment, Methods and Tools, Requirements, Engineering Designer

\section{Introduction}

This paper is partly based on the author's recently presented doctoral thesis titled "Engineering Designers' Requirements on Design for Environment Methods and Tools" [1]. In this paper, however, the author's previously presented research has been strengthened with empirical data from three new studies by Jilderin and Omberg [2] and Rilegård [3] $]^{1}$.

\footnotetext{
${ }^{1}$ Presents two studies, one interview and one questionnaire study.
}

\section{Objectives}

The objectives of this paper are twofold:

- Identify engineering designers' utilization of DfE methods and tools; and

- investigate what design-related requirements a DfE method or tool should fulfill in order to become actively used ${ }^{2}$ in industry among engineering designers.

\section{Design for Environment (DfE)}

Design for Environment (DfE) has become more and more important for companies in recent years. Bakker [4] defines DfE as "the development of products by applying environmental criteria aimed at the reduction of environmental impact along the stages of the product's life cycle".

There has been a trend towards the rapid development of DfE methods and tools in recent years, with the result of a considerable number of new DfE methods and tools as seen in, for example, Simon et al. [5], Wrisberg et al. [6] and Ernzer and Birkhofer [7]. Potential DfE methods and tools fall into a wide range of categories, from relatively simple checklists or general guidelines to more complex software-based decision-making methods [6].

According to Mathieux et al. [8], extensive research on DfE has mainly been carried out by research organizations and industrial companies. A great amount of relevant literature has been published concerning how to perform DfE, for example Brezet and Gertsakis et al. [9]. The ISO has developed a standard within the ISO 14000 framework on how to integrate environmental aspects into product development [10].

\footnotetext{
${ }^{2}$ Actively used is defined as "when a method or tool is regularly utilized because of a high degree of appropriateness and usability”. Usability is defined as "the support to solve a specific task with efficiency (to do things right ) and effectiveness (doing the right things)", and Appropriateness as "the user's integrated comprehension of the quality of the outcome of the method or tool" [1]
} 


\subsection{Use of DfE}

Ritzén and Lindahl [11] as well as Ernzer and Birkhofer [7] state that despite the number of available DfE methods and tools, there are relatively few which are widely used by companies. One reason for this low utilization is that they are time-consuming; another is that many of them focus on only on environmental issues [12]. For the enterprises and their engineering designers ${ }^{3}$, of course, the environmental issue is but one of many issues to be considered.

When DfE methods and tools are used, these methods and tools are often not integrated in the product development process. This is a point highlighted by Baumann et al. [13], Ernzer and Birkhofer [7] and Tukker et al., [14] and was also a finding of the author in his earlier research $[15,16]$. NUTEK, the Swedish Business Development Agency [17], had a similar conclusion in its final report on a three-year-long DfE project.

\subsection{Isolated development of DfE methods and tools}

Even though more and more approaches focus on how to perform DfE, as well as on what is required for its successful integration, there seems to be a gap between the developers and the presumptive users. Bauman et al. [13] have examined articles ${ }^{4}$ concerning methods and tools in the area of DfE. They found that there were relatively few references describing the diffusion of DfE methods and tools, the experience of how these tools and methods worked in product development, and how useful these methods were in actually reducing the environmental impacts of products. Baumann et al. conclude that most publications with an empirical content report on the testing of new DfE methods and tools, and that these are often developed at universities and tested by researchers in company case studies. This is supported by Tukker et al. [14]. They report that many of the DfE methods and tools are developed by researchers within universities or research institutes. In some cases, there is little or no testing of these methods and tools in industrial practice.

Based on their literature review, Baumann et al. [13] conclude that there has been an overabundance of DfE method and tool development. According to these authors, the references indicate that those involved in the field are more interested in developing new DfE methods and tools, rather than in studying the use of existing ones in order to evaluate and improve them, as well as evaluat-

\footnotetext{
3 "Engineering designers" are herein referred to as "designers"

${ }^{4}$ Their literature study is based on a cross-disciplinary database containing around 650 research articles, taken from the engineering, management, and policy studies disciplines.
}

ing them in accordance with how well they fit into product development.

\subsection{The designer - a presumptive key user}

To achieve a successful product development effort, it is important to make the right decisions from the beginning in order to avoid expensive changes and delays. It is also important to select appropriate methods and tools and, at an early stage, to involve needed competencies [18]. These competencies, in turn, influence methods and tools selection, and vice versa. Designers, i.e. those who are involved in giving the product a design (such as design engineers and industrial designers) are a central competence group in product development. Therefore, the position taken is that designers bring crucial competencies to successful DfE initiatives, and should therefore be important primary presumptive and practical users of DfE methods and tools.

If the ambition is to integrate DfE into ordinary product development - as is the ambition with ISO 14046 [10] - then there is also an essential need to involve and consider one of the main presumptive users of DfE methods and tools: the designer. In fact, it is the designer who is often the main practical executer of methods and tools used to develop a product. Even if designers do not always decide what method or tool to use, their use influences the outcome and the benefits from the use. When using a method or tool, it is important to understand its various advantages and disadvantages. It is also important to know under what circumstances the method or tool's result is valid. This implies that a method or tool is dependent on the user, i.e. whether the user misuses or does not understand how to use the method or tool, or whether the result and/or interpretation of the result will reflect this [19]. The method or tool user always has interpretation precedence. It is more or less irrelevant whether the aim of the method or tool developer was to emphasize environmental impact if the user does not realize that, but instead utilizes the method or tool for other reasons, such as, for example, to save costs. In short, what the user interprets is what matters the most.

\section{$4 \quad$ Utilization and usefulness}

According to Ritzén [20], the usage of methods and tools only becomes a regular activity if they support the users, in this case designers, with their own work. Considering the above in combination with the low level of industry utilization of DfE methods and tools, a developer of DfE methods and tools should consider why DfE methods and tools have such limited use in industry [1]. One possible explanation could be that the DfE method or tool 
does not fulfill the users' - i.e. designers' - requirements ${ }^{5}$. If so, the application of those requirements could be useful for further development of the DfE method or tool. This paper deals with the identification of those designer requirements.

Focusing solely on utilization when evaluating different methods and tools may be dangerous. There is a major risk that the wrong conclusions will be drawn, for example what requirements a method or tool should fulfill in order to be regularly utilized. Just because a designer regularly utilizes a method or tool does not necessary imply that method or tool is successfully applied. One example of this is when the utilization is a formal must in the product development process, and the designer considers the outcome useless, already known or not needed for the further product development work.

Another example of when only focusing on utilization is dangerous is when a method provides a useful outcome for a product that is valid for an extended period, perhaps for several product generations. This could imply that the designer does not comprehend any need to utilize the method regularly, even though the outcome is useful ${ }^{6}$. The low or non-existing utilization could also be a result of management issues that prevent the utilization, or because the method or tool is unknown to the designers.

When examining different methods and tools, it important to note and be aware of the fact that their major purposes may differ substantially. For example, the purpose of brainstorming differs greatly from that of various types of DfE tools. Sometimes, the main purpose with the use of the method or tool is not the outcome itself, but rather how the outcome is received.

It is the author's conclusion that general methods and tools, as well as DfE method and tool utilization, must be studied and related to the overall context of the situation, for example the type of product development or level of education $^{7}$ [1]. Utilization is just a quantitative measure of the frequency of use. It is related to the number of product development projects, and is a function of its usefulness, i.e. whether the method or tool suits the purpose. From a designer's perspective, the usefulness of a method or tool depends on two different parameters (even if interlinked): the quality of the method or tool and the quality of the outcome. Both these two parameters are partly subjective and context-related quality measures, implying that they can be difficult to measure scientifically.

\footnotetext{
${ }^{5}$ Requirement is in this paper defined as "a specific description of an attribute".

${ }^{6}$ However, with this type of utilization, there is a risk that the designer will rely on a previous outcome and his changes imply that the outcome is not valid any longer. In a way, changes will always imply that the outcome changes, but the problem is to know when there is a need for a new updated version.

${ }^{7}$ See also Riztén and Lindahl [11].
}

In this paper, the concept usability is introduced to describe the user's integrated comprehension of the userelated qualities of the method or tool, for example if they are easy to learn and use. Other qualities are the method or tool's support concerning efficiency, as in "to do things right", and effectiveness, as in "doing the right things" [21]. Given this, usability is defined as "the support to solve a specific task with efficiency and effectiveness". High usability refers to when a tool enables its users to generate an outcome in the early phases of the product development process.

Another concept used is appropriateness: the user's integrated comprehension of the quality of the outcome of the method or tool. The quality of the outcome is a combination of factual and subjective aspects, is highly context-related, and is where the satisfaction of the outcome influences the degree of appropriateness. An example of a factual aspect could be that the outcome accuracy is within the limits defined by the customer, while a subjective aspect could be that the user does not consider the outcome reliable. The two different parameters imply that four major types of method and tool usefulness exist, as shown in Figure 1.

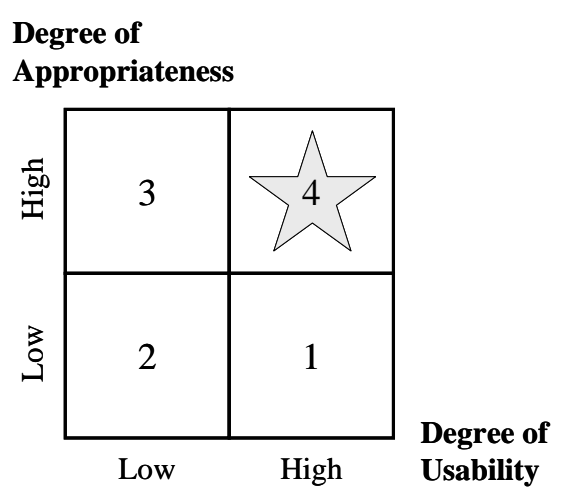

Figure 1. The principal relation between a method or tool's degree of appropriateness and its usability. Degree of usability is related to a method or tool's support to solve a specific task with efficiency and effectiveness. Degree of appropriateness describes the user's integrated comprehension of the quality of the outcome of the method or tool. The quality of the outcome is a combination of factual and subjective aspects, is highly context-related, and is where the satisfaction of the outcome influences the degree of appropriateness [1].

The first type is when a method or a tool has a high degree of usability, but the designer experiences the gains or appropriateness as low. An occasion when this could be the case is when the use of a method is dictated as a formal "must" in the product development process. Nevertheless, the usability of the outcome for the individual 
designer is low, for example because the outcome is already known or not needed for further work.

The second type is when a method's low usability results in a poor understanding of the method's benefits, and thus low appropriateness.

In the third type, the designer's comprehension of the usability is low, but they consider the outcome as having a high degree of appropriateness; one example is a method or tool that is very tricky and complicated to learn and use, but where the outcome compensates for this.

The fourth type is the ideal one, and an example of high usability and appropriateness. For example, a designer regularly utilizes a CAD program whose outcome gains the concept of his or her own ideas - a concept that implies further utilization such as an increased degree of usability and appropriateness. This usability and appropriateness, in turn, generates increased utilization. This type of utilization is denoted in this paper as actively used, and defined as "when a method or tool is regularly utilized because of a high degree of appropriateness and usability".

\section{Research method}

A major challenge for this research has been how to study DfE methods and tools, when they are so rarely utilized within the industry. This section explains the general research strategy to solve this problem and the specific research strategy selected here.

Research on environmental issues highlights some risks, for example the fact that people tend to respond to what is assumed as morally good or politically correct in a specific culture/context. This risk, in combination with the low use of DfE methods and tools, was expected to make it hard to exclusively study these kinds of methods and tools. Instead, in order to obtain a wider perspective on designers' use of methods and tools, and to avoid negatively influencing the respondents, the focus has been on the general use of methods and tools. This wider perspective is based on the assumption that the basic requirements for any method or tool to become utilized are basically the same. "Requirement" is, in this context, defined as a "specific description of an attribute of something”.

The unit of analysis is the designer's experience of used methods and tools. Investigating designers' own experience of methods and tools is a means for finding industrially applicable methods and tools, i.e. DfE methods and tools. Designers' requirements for methods and tools can be empirically investigated in several ways; in this research, the following two ways have been used:

- Directly, by asking designers what requirements they have for their methods and tools.
- Indirectly, by asking designers about their experiences with utilized methods and tools, in order to find characteristics that unite these methods and tools and make them easier to explain characteristics that can later be transformed into requirements.

Based on the research strategy, two types of research methods have been used: qualitative research interviews and questionnaire studies. Altogether, this paper is based on five qualitative research interview studies and two questionnaire studies (see Table 1). All participating designers are from Swedish companies.

Table 1. Number of respondents and participating companies in the research studies. I - "Experience of and requirements on methods for product development" [19], II "Engineering designers' experience of design for environment methods and tools" [22], III - "Usability and demands on design engineering tools and methods utilized in Small and Medium Sized Enterprises" [2], IV - "Use and perception of Design for Environment (DfE) in Small and Medium Sized Enterprises in Sweden" [23], V-VI - "Criteria for good design for environment methods and tools - a study of small and medium size enterprises requirement" [3] and VII - "User Requirements for Design for Environment methods and tools" [24].

\begin{tabular}{|c|c|c|c|c|c|c|c|}
\hline Study: & I & II & III & IV & $\mathbf{V}$ & VI & VII \\
\hline Туре & \multicolumn{5}{|c|}{ Interview } & \multicolumn{2}{|c|}{ Questionaire $^{8}$} \\
\hline Companies & 1 & 1 & 5 & 10 & 7 & $14^{9}$ & 24 \\
\hline Departments & 1 & 3 & 5 & - & - & - & - \\
\hline Respondents & 11 & 13 & 10 & 15 & 9 & 62 & 203 \\
\hline Interview time ${ }^{10}$ & \multicolumn{5}{|c|}{1 hour } & & \\
\hline Responserate & & & & & & $77 \%$ & $96 \%$ \\
\hline
\end{tabular}

\section{Discussion}

The studies reveal that designers view themselves as users of methods and tools in quite low numbers, which the analysis of the results from the questionnaire emphasizes [1]. The most utilized tools are various kinds of Computer-Aided Design (CAD) tools; if all CAD tools are considered as one tool, then there exist few additional methods and tools utilized independently by more than a fifth of the respondents [24]. Among DfE relatedmethods and tools, the only ones mentioned during the studies have been EEA and LCA. In the study presented

\footnotetext{
${ }^{8}$ Both questionnaires were Internet-based and distribuated electronically via e-mail. Several questions were logical and dynamic questions, meaning that one answer influenced further questions.

${ }^{9} 8$ major and 16 small and medium-sized enterprises.

${ }^{10}$ The average interview time per respondent.
} 
in [24], EEA was used by respondents from the majority of the companies, but LCA appeared to be used at very few companies. Aside from two exceptions, the respondents that used LCA were all working at major companies, with half of these respondents at the same company. It is interesting to note that EEA was so widespread among so many different companies. It seemed more probable that LCA would be more common among the companies, due to the major focus on that method in Sweden and the fact that the method is older than the EEA method.

Based on the research, it was not possible to conclude whether this result was based on the fact that EEA was developed from on lessons learned in the use of other DfE methods and tools, i.e. designer requirements on a method or tool [25]. Nevertheless, it has most likely had some influence.

\subsection{Designers' method and tool requirements}

Klein [26] states that a democratic or participative method or tool needs to include what is important to the people in the situation, even if this does not match the original purpose defined by the method or tool developer. The author's conclusion is that it is important to understand the context in which a method or a tool is used in order to state requirements on the method or tool. Based on studies by Norell [18], Klein [26], Ehrenfeld and Lenox [12], Ritzén [20] and Ernzer and Birkhofer [7], the conclusion is further strengthened. The research has highlighted that this understanding of the context in which designers use methods and tools seems to be an important issue.

The above conclusion, especially considering Klein [26], implies that DfE methods and tools must not only be appropriate from an environmental perspective, but from a user perspective as well. Further, if a method or tool is developed and applied without understanding the circumstances in which it was developed and without a diagnostic approach to the circumstances in which it is to be applied, then the likely result will be inappropriate application.

The discussion above implies that whether a method or tool is actively used or not does not simply depend on the method or tool itself. As shown in the studies, a method or tool can be actively used in one context but not in another, something which is also supported by Ernzer and Birkhofer [7]. When a method or tool is selected and later implemented, several context-related aspects, for example organizing arrangements, social factors, physical settings, visions, and technology, influence their active use as well as the existing context. NUTEK's [27] conclusion is in line with this, as it states that the company's size, branch, operations and even the expertise that exists within the company are all important to the best-suited choice of method and tool. Change in one factor will usually require complementary changes in another. This implies that the interdependence between organizational characteristics must be taken into account.

\section{Discussion about identified method and tool requirements}

Despite the lack of identified formal requirements in the studies, the designers had several informal requirements, often connected with the reasons expressed for utilizing methods and tools. This section presents and briefly discusses the method and tool requirements identified from this research, several of which are interlinked.

\section{Appropriateness-related requirement}

Reliable and relevant outcome - One of the most important requirements is that a method or tool must provide outcomes that are relevant and reliable for the user, in this case the designer. The requirement itself is problematic depending on the problem described, especially when considering how to quantify what an appropriate outcome is. In other words, what for one designer may be considered a reliable and relevant outcome may not be for another. For DfE methods and tools, where are often problems in finding adequate data, the issue of reliable and relevant outcomes becomes even more difficult since much of the used data is instead based on validations, assumptions and limitations. The capacity to validate whether an outcome is reliable and relevant is dependent on the user's experience and knowledge. As shown in the studies, designers' knowledge about DfE-related issues i.e. their capacity to validate the outcome - appears to be low.

\section{Usability-related requirements}

Direction towards a target area rather than a road map to the target - This requirement relates to the method or tool's degree of usability, i.e. efficiency and effectiveness. "Freedom of action" appears, at least according to this research, to be important for the designers. It also seems probable that this is the very nature of the product development, often called the design paradox ${ }^{11}$ [28].

Time efficient - This was not only one of the most frequently mentioned requirements during the studies, but is also a more or less explicitly mentioned requirement in the literature. The requirement could be interpreted as a logical consequence of increased competition [29] and the design paradox. The requirement is related to the degree

\footnotetext{
${ }^{11}$ The paradox is that when the general design information is needed, it is not accessible, and when it is accessible, the information is usually not needed [1].
} 
of usability, i.e. to "do things right" and to "do the right things” (see Section 4).

Setup time - Related to time efficiency is the conclusion based on the studies that a method or tool must not have an excessive setup time. For designer comprehension, complicated methods and tools must be daily or at least regularly utilized; otherwise, designers tend to forget how to utilize the method or tool's specific functions and the setup time increases ${ }^{12}$. At the same time, this implies that the number of methods and tools a designer can utilize is limited.

Not require excessive simultaneous collaboration Collaboration takes time and coordination, influences the setup time and diminishes designers' freedom of action [1].

Integration - Adjustable to different contexts Mentioned more or less explicitly by some designers during the studies is the different companies' culture or specific ways of performing tasks, often a fundamental reason for the success of a company. This implies that a method or tool must be able to fit into and be adjustable to this culture, i.e. the specific context, an assertion also supported by Ernzer and Birkhofer [7]. Further, if the method or tool is not sufficiently integrated or adjusted into a company's context, there is also a risk for standalone methods and tools as described by Ehrenfeld and Lenox [12], i.e. methods and tools with outcomes not appropriate to designers. During the inverview studies, respondents expressed several examples of stand-alone methods and tools.

Computer based - When looking at which methods and tools are used more regularly, the conclusion based on this reasearch and other existing research studies such as e.g. Norell [18] is that the method or tool should be computer-based.

Easy to adopt and implement - This can be seen as an overarching requirement that can be divided into subrequirements:

- Gradually introduced gadgets/functions - A method or tool must not be experienced as being too complex/complicated, at least not from the beginning. This implies that the user should not be required to know everything and understand the method or tool's functions from the beginning to be able to use them. When the user gains experience, he or she can add more and more gadgets/functions to make, for example, the result more and more precise. According to many of the respondents in the studies, too many methods and tools attempt to accomplish too much from the beginning instead of starting at a lower level. According to some of the designers in the studies, it is better to have many different methods or tools that

\footnotetext{
${ }^{12}$ For further discussion, see Lindahl [22].
}

complement each other rather than one that tries to cover everything, especially when only a part of the method or tool is used.

- Easy to understand the benefits - Simplicity also requires that it must be easy to understand and experience the benefits of the method or tool, something which is in line with Norell [18]. According to the inverview studies, the method or tool must be intuitive, logical and easy to communicate, and the benefits received by applying the method or tool must be obvious in comparison to the effort that would be needed if the method or tool was not used. This requirement is especially important for rarely used methods and tools.

- Intuitive and logical - The presumptive user ought to be able to realize how the method or tool and its different parts fit together. Designers in the studies understand that it is advantageous if the method or tool is intuitive and resembles other methods or tools that have been used. This may provide benefits such as a faster learning process and easier integration of different methods and tools. For example, if the designers are already familiar with FMEA, this seems to aid the learning and intuitive understanding of the methodically-similar EEA method [30]. On the other hand, if the designer must read several hundred pages of a long description or manual (often with academic jargon) before even using the method or tool, it is likely that he or she never will even start reading. Instead, designers in the inverview studies preferred methods and tools requiring a minimum of education, and which they could start up and use immediately. After that, if questions occur, they should know where to find answers, for example from a manual. The conclusion is that the intuitive and logical aspect is especially important for methods and tools that are rarely used.

\subsection{Method and tool requirements' implications on DfE methods and tools}

If DfE methods and tools are to become actively used ${ }^{13}$ in the product development process, it is important to increase the understanding about what make users consider them as usable and appropriate. Whether a DfE method or tool has a specific environmental degree of appropriateness is more or less irrelevant unless it is not utilized.

The studies have generated several requirements that can be adopted for the further development of DfE methods and tools.

\footnotetext{
${ }^{13}$ The opposite would imply that there were no need in the first place to develop something neither needed nor asked for.
} 


\section{Conclusions}

The conclusion is that the new studies by Jilderin and Omberg [2] and Rilegård [3] support and strengthen the earlier conclusions drawn in Lindahl [1].

Designers' utilization of DfE methods and tools is limited. Depending on the context, different actors' requirements are more or less important. In this research, the focus has been on designers' requirements, since they are considered as key users because of their strong influence on utilization and the quality of the outcome. The conclusion is that it is more or less impossible to discuss requirements for a method or tool unless considering the context in which the method or tool will be utilized. Further, the major actor involved must gain something, for example a more time-efficient product development, from using the method or tool unless it is likely that the utilization will stop or perhaps never even start. As this research has shown, the major actor in the product development is the designer.

Of all the designer-related requirements given and validated by the respondents, most are related to designers' aims to fulfill the product performance and keep down the development time. This can be summarized into four major requirements, of which three are interlinked. The conclusion is that a DfE tool as well as a common method or tool must exhibit the following:

1. Be easy to adopt and implement. Whether a method or tool fulfills the three following requirements is of lesser importance if leads to problems with adoption and implementation and is seen as having a low degree of usability, and therefore is not utilized by the designers in their daily work. This requirement is the key for a method or tool to become actively used.

2. Facilitate designers to fulfill specified requirements on the presumptive product and at the same time...

3. ...reduce the risk that important elements in the product development phase are forgotten.

Both of these two latter requirements relate to a method or tool's degree of appropriateness. The second and the third requirements are related to the fourth requirement, which is considered by the author to be the most important:

4. The use of the method or tool must reduce the total calendar time (from start to end) to solve the task. If the method or tool helps designers to fulfill specified requirements, it will also most likely help them to reduce the calendar time as well as the number of working hours needed to develop the product. This is also something that enables designers to introduce changes in early phases of the product development process when changes still are easy to make. Likewise, if the method or tool reduces the risk that important moments in the product development are forgotten, it will most likely have a positive effect and reduce the calendar time and number of working hours needed.

Of course, other requirements can be added and the above four can be divided into more detailed requirements. However, much would be gained if these four requirements were used as a first overall validation of the usefulness of the method or tool. If the result of the validation is positive, further requirements that are more detailed can be used in order to obtain a more detailed validation.

\section{Acknowledgement}

The author the paper would like to acknowledge Johan Jilderin, Henrik Omberg and Erik Rilegård for their support in the empirical data collection.

\section{References}

[1] Lindahl, M. (2005), Engineering Designers' Requirements on Design for Environment Methods and Tools, in Industrial Engineering and Management. KTH: Stockholm, Sweden.

[2] Jilderin, J. and H. Omberg (2004), Usability and demands on design engineering tools and methods utilized in Small and Medium Sized Enterprises - A different approach to understand the reasons behind the low utilization of DfE methods and tools. University of Linköping: Linköping, Sweden.

[3] Rilegård, E. (2005), Criteria for good design for environment methods and tools - a study of small and medium size enterprises requirement (Swedish only). Environmental Technology and Management, Department of Mechanical Engineering, Linköping University: Linköping, Sweden.

[4] Bakker, C. (1995), Environmental Information for Industrial Designers, in PhD-Thesis. Delft University of Technology: Delft, Holland.

[5] Simon, M., S. Evans, T.C. McAloone, A. Sweatman, T. Bhamra, and S. Poole (1998), Ecodesign Navigator - A Key resource in the Drive towards Environmentally Efficient Product Design. Manchester, UK: Manchester Metropolitan University, Cranfield University \& EPSRC.

[6] Wrisberg, N., H.A. Udo de Haes, U. Triebswetter, P. Eder, and R. Clift (2000), ed.^eds. Analytical Tools for Environmental Design and Management in a Systems Perspective. Kluwer Academic Publishers: Dordrecht.

[7] Ernzer, M. and H. Birkhofer (2002). Selecting Methods for Life Cycle Design Based on the Needs of a Company. in International Design Conference - Design 2002. Dubrovnik, Croatia.

[8] Mathieux, F., G. Rebitzer, S. Ferrendier, M. Simon, and D. Froelich (2001), Ecodesign in the European Electr(on)ics Industry - An analysis of the current practices based on cases studies - An analysis of the current practices based on cases studies. The Journal of Sustainable Product Design, 1(4): p. pp. 233-245. 
[9] Gertsakis, J., H. Lewis, and C. Ryan (1997), A Guide to EcoReDesign - Improving the Environmental Performance of Manufactured Products. Melbourne, Australia: Centre for Design at RMIT.

[10] ISO 14062 (2002), Environmental management Integrating environmental aspects into product design and development. International Organization for Standardization: Geneva, Switzerland. p. 24.

[11] Ritzén, S. and M. Lindahl (2001). Selection and implementation - key activities to successful use of EcoDesign tools. in Proceedings EcoDesign 2001: Second International Symposium on Environmentally Conscious Design and Inverse Manufacturing. Tokyo, Japan.

[12] Ehrenfeld, J.R. and M.J. Lenox (1997), The Development and Implementation of DfE Programmes. The Journal of Sustainable Product Design., (1): p. 17-27.

[13] Baumann, H., F. Boons, and A. Bragd (2002), Mapping the green product development field: engineering, policy and business perspectives. Journal of Cleaner Production, 10(5): p. 409-425.

[14] Tukker, A., E. Haag, and P. Eder (2000), Eco-design: European state of the art - Part I: Comparative Analysis and Conclusions - An ESTO project report. European Commission Joint Research Centre Institute for Prospective Technological Studies: Brussels, Luxembourg. p. 60

[15] Ernzer, M., M. Lindahl, K. Masui, and T. Sakao (2003). An international study on Utilization of Design for Environment Methods (DfE) - a pre-study. in EcoDesign 2003: 3rd International Symposium on Environmentally Conscious Design and Inverse Manufacturing. Tokyo, Japan: Union of EcoDesigners (Association of EcoDesign Societies, Japan).

[16] Lindahl, M. (2003). Designer's utilization of DfE methods. in Proceedings of the 1st International Workshop on "Sustainable Consumption". Tokyo, Japan: The Society of NonTraditional Technology (SNTT) and Research Center for Life Cycle Assessment (AIST).

[17] Nutek (2002), Metodik för Miljöanpassad produktutveckling i små och medelstora företag - slutrapport från ett treårigt NU-TEK-program (Methodology for Design for Environment in small and medium sized enterprises - Final report from a three-year Nutek-program) - In Swedish. Nutek: Stockholm, Sweden.

[18] Norell, M. (1992), Stödmetoder och samverkan i produktutveckling, in Department of Machine Elements. Royal Institute of Technology: Stockholm, Sweden.

[19] Lindahl, M. and A.-M. Åkermark (2004), Experience of and requirements on methods for product development - An interview survey at a major Swedish vehicle company: Linköping, Sweden.

[20] Ritzén, S. (2000), Integrating environmental aspects into product development - Proactive measures, in Integrated Product Development Division, Dept. of Machine Design. Royal Institute of Technology: Stockholm, Sweden.
[21] Hill, T. (1995), Manufacturing Strategy, Text and Cases. London, UK: MacMillan Press Limited.

[22] Lindahl, M. (2005), Engineering designers' experience of design for environment methods and tools - Requirement definitions from an interview study. Journal of Cleaner Production, Elsevier, In press.

[23] Lindahl, M., L. Skoglund, J. Svensson, and R. Karlsson (2003). Use and perception of Design for Environment (DfE) in Small and Medium Sized Enterprises in Sweden. in EcoDesign 2003: 3rd International Symposium on Environmentally Conscious Design and Inverse Manufacturing. Tokyo, Japan: Union of EcoDesigners (Association of EcoDesign Societies, Japan).

[24] Lindahl, M. (2004), User Requirements for Design for Environment methods and tools - Based on a web-based questionnaire survey. Journal of Sustainable Product Design, Submitted for publication.

[25] Lindahl, M. (2001). Environmental effect analysis - how does the method stand in relation to lessons learned from the use of other design for environment methods. in Proceedings EcoDesign 2001: Second International Symposium on Environmentally Conscious Design and Inverse Manufacturing. Tokyo, Japan.

[26] Klein, L. (1994), Sociotechnical/organizational design, in Organisation and Management of Advanced Manufacturing, W. Karwowski and G. Salvendy, Editors. John Wiley \& Sons Inc.: New York, US.

[27] Nutek (2003), Sustainable products - new business through Design for Environment. Swedish Business Development Agency: Stockholm, Sweden.

[28] Ullman, D.G. (1997), The Mechanical Design Process. 2nd ed. New York: McGraw-Hill.

[29] Stalk, G.J. and T.M. Hout (1990), Competing Against Time - How Time-Based Competition is Reshaping the Global Markets. New York, USA: The Free Press, A Division of Macmillan Inc. 285.

[30] Lindahl, M. (2000), Environmental Effect Analysis - an approach to design for environment, in Dept. of Chemical Engineering and Technology. Royal Institute of Technology: Stockholm, Sweden. 